\title{
Datenschutzfalle Praxismonitor
}

\author{
PC-Monitore in der Arztpraxis bergen eine oft unterschätzte Gefahr: Die Bildschirmdaten lassen sich auch \\ von der Seite einsehen. Privacy-Filter können das Datenschutzproblem beheben.
}

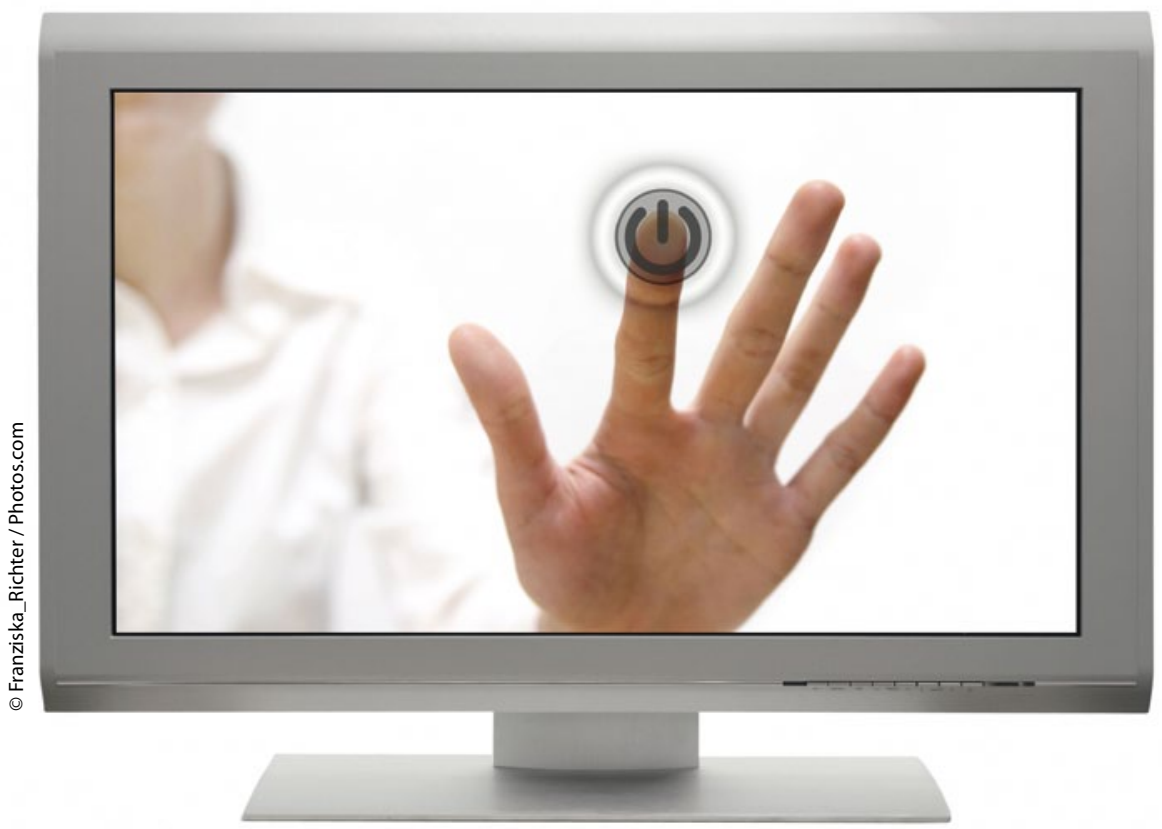

$\longrightarrow$ erade wurde die Praxis geöffnet, schlängeln sich auch schon die ersten Patienten um die Anmeldung. Und selbst wenn diese zunächst noch einen gewissen Diskretionsabstand halten, lehnen sich wenn sie an der Reihe sind schnell über dem Tresen. Keine Chance für die MFA die Daten des vorhergehenden Patienten am PC zu schließen. Da hilft nur eines, um den strengen Datenschutzanforderungen an die Praxis gerecht zu werden: aufrüsten - egal ob baulich oder technisch.

\section{Risikofaktor offen gestaltete Räume}

Die Bundesärztekammer und die Kassenärztliche Bundesvereinigung schreiben in ihren „Empfehlungen zur ärztlichen Schweigepflicht, Datenschutz und Datenverarbeitung in der Arztpraxis" ganz klar vor, dass der Arzt gewährleisten muss, „dass sowohl im Empfangsbereich als auch in den Behandlungsräumen unbefugte Dritte keinen Zugriff (Einblick) in die Patientendaten erhalten " dürfen. Bildschirme seien so aufzustellen, dass sie nur vom Arzt und dem Praxispersonal eingesehen werden können. Doch das ist gerade bei modernen Tresengestaltungen etwa mit Rundtresen, die die Räume optisch auflockern sollen, aber auch bei Tresen, die um die Ecke gehen, nicht so einfach. Abhilfe können Tresenerhöhungen oder kleine Umbauten um den PC-Bildschirm schaffen. Wer auf Nummer sicher gehen will, nutzt - am besten in Kombination - folgende Varianten: einen Sichtschutzfilter für den PC-Bildschirm sowie einen passwortgeschützten Bildschirmschoner, der sich automatisch einschaltet, wird der Rechner länger nicht genutzt. Letztere Variante sollte auch im Behandlungszimmer genutzt werden.

\section{Schutzfilter reduziert \\ Einsichtswinkel auf $30 \mathrm{Grad}$}

Im Behandlungszimmer, wo Patienten nur einzeln Zutritt erhalten, könnte auch gänzlich auf den automatischen Bildschirmschoner verzichtet werden und dafür - warten Patienten länger alleine im
Sprechzimmer auf den Arzt - der Rechner per Hand gesperrt werden, bevor ein neuer Patient den Raum betritt.

Heikel ist das Thema Datenschutz am Empfang, weil hier mehr Augen um den Bildschirm herum versammelt sind. In diesem Bereich könnte zusätzlich auf die Sichtschutzfilter oder Privacy Filter zurückgegriffen werden. Hierbei handelt es sich um eine Art Kunststofffolie, die mithilfe von kleinen Haltelaschen, beidseitig klebenden Stickern oder zum Teil Rahmen in der Farbe des Monitors auf dem Bildschirm angebracht wird. In der Regel reduzieren diese Filter den Einsichtswinkel des Bildschirms von den Seiten und von oben auf 30 Grad - sodass Patienten, die am Tresen stehen, kaum die Möglichkeit haben, Daten am Bildschirm zu erkennen.

\section{Zusatznutzen entscheidet}

Die Privacy Filter gibt es für fast jede Bildschirmgröße, und der Anschaffungsaufwand hält sich auch in Grenzen. So kostet der Hewlett-Packard (HP) 14-Zoll Privacy Screen rund 56 EUR. Aber auch die Varianten für 19-Zoll-Monitore, die in den Praxen gängiger sein dürften, sprengen nicht gleich das Budget: Der Kensington LCD-Sichtschutzfilter 19-Zoll liegt etwa bei 85 EUR, der 15-Zoll Laptop-Sichtschutzfilter bei 61 EUR. Etwas günstiger ist der 19-Zoll Targus Privacy Filter, der 79,90 EUR kostet. Für den 3M Privacy Filter 15,6-Zoll müssen Praxen rund 85 EUR zahlen. Es gibt aber auch von hsw3000 (4ProTec Blickschutz), Griffin, Dicota und Crumpler Sichtschutzfilter. Dabei gewähren alle Filter einen Einsichtswinkel von 30 Grad, die Wahl des richtigen Filters hängt also eher von Preis und Zusatznutzen ab. Einige Filter reduzieren nämlich gleichzeitig auch die Blendwirkung und die statische Aufladung des Bildschirms und damit die Staubablagerung. Schaden kann es zudem nicht, wenn auf ISO-Standards und das Erfüllen von Arbeitsschutzrichtlinien geachtet wird.

RebekkaHöh 